\title{
Lapurdum
}

Euskal ikerketen aldizkaria | Revue d'études basques |

Revista de estudios vascos | Basque studies review

$11 \mid 2006$

Numéro XI

\section{Les revendications institutionnelles contemporaines en Pays basque de France : représentations du monde basque et discours politiques}

\section{Thomas Pierre}

\section{(2) OpenEdition}

1 Journals

Édition électronique

URL : http://journals.openedition.org/lapurdum/408

DOI : $10.4000 /$ lapurdum.408

ISSN : 1965-0655

Éditeur

IKER

Édition imprimée

Date de publication : 1 novembre 2006

Pagination : 291-303

ISBN : 978-2-86781-474-7

ISSN : $1273-3830$

Référence électronique

Thomas Pierre, « Les revendications institutionnelles contemporaines en Pays basque de France: représentations du monde basque et discours politiques », Lapurdum [En ligne], 11 | 2006, mis en ligne le 01 avril 2008, consulté le 03 mai 2019. URL : http://journals.openedition.org/lapurdum/408 ; DOI : 10.4000/lapurdum.408 


\section{Les revendications}

institutionnelles contemporaines en Pays basque de France : représentations du monde basque et discours politiques

Thomas PIERRE

Doctorant en Anthropologie sociale et Ethnologie - LAIOS - EHESS - Paris

En Pays basque de France, la revendication institutionnelle - dans son incarnation départementale - s'est manifestée sous de multiples tendances depuis la Révolution Française. De nombreux épisodes marquent sa continuité relative sous des formes différentes. L'appellation départementale n'est en effet récurrente que dans la mesure où elle a pour objectif la reconnaissance du Pays basque nord par le biais d'une institution territoriale dans le cadre politico-administratif français.

Entre l'époque révolutionnaire et le milieu du XIX ${ }^{\mathrm{e}}$ siècle, la revendication réapparait régulièrement mais les caractéristiques des représentants et/ou des différents acteurs oscillent largement entre un discours économiste - sous l'impulsion des milieux issus de la Chambre de Commerce et d'Industrie de Bayonne - et un discours à tendance ethno-culturelle - sous les vocables utilisés par les représentants des institutions locales pré-révolutionnaires. Puis, à partir des années 1960, viennent les versions modernes de la revendication. À cette époque, le mouvement indépendantiste Enbata inscrit dans son programme politique la revendication de création d'un Département Basque. 
Depuis cette époque, deux groupes d'acteurs militent pour la reconnaissance politique de l'identité basque par l'institutionnalisation départementale du Pays basque de France. Le premier groupe rassemble les différentes tendances abertzale (nationaliste basque) et le mouvement culturel basque. Le second, les élus locaux non-nationalistes mais basquisants et les milieux proches de la Chambre de Commerce et d'Industrie de Bayonne, pour laquelle la reconnaissance territoriale doit contribuer au développement économique du Pays basque français. Jusqu'à la fin des années 1980, face aux diverses résurgences du mouvement, l'État répond par quelques réformes administratives dont l'objectif central est d'exclure l'hypothèse d'une division du département des Pyrénées-Atlantiques par la création de deux départements distincts, l'un Pays Basque, l'autre Béarn. Cette politique permet à l'État de se limiter à quelques concessions et à tempérer les effets de la dynamique départementaliste et nationaliste ${ }^{1}$.

\section{La tentative étatique de gestion des aspirations culturelles basques}

Néanmoins, dans le courant des années 1990, le gouvernement s'inquiète du renouveau des aspirations basquisantes. Il tente alors de contrôler la contestation portée par les abertzale en la redéfinissant comme un problème de développement local et non plus comme une question institutionnelle. L'objectif est de décrédibiliser la revendication institutionnelle, en l'occurrence, départementaliste.

Une démarche de prospection territoriale, intitulée " Pays Basque 2010 », est lancée. Le sous-préfet invite alors les élus politiques, les représentants de la société civile, ainsi que les différents services publics, à entreprendre une réflexion sur l'avenir du territoire « Pays Basque » français à l'horizon 2010.

La référence au territoire doit permettre de reconnaître la légitimité des aspirations basquisantes tout en les intégrant dans un nouveau cadre d'action publique.

Il s'agit, par l'intermédiaire du concept de territoire "Pays Basque ", de donner un sens nouveau à l'identité collective. Par le biais du concept de territoire, la notion d'identité est alors reliée à la sphère économique.

L'État tente en effet d'apporter une réponse à la revendication de reconnaissance identitaire portée par les différentes sphères basquisantes - les abertzale, les départementalistes et le mouvement culturel basque - par la création de deux conseils (le Conseil des Élus et le Conseil de développement du Pays Basque) qui doivent permettre de créer les cadres adaptés au développement d'un échange politique serein entre l'État, la classe politique locale et les mouvements basquisants. Dans ce contexte, la culture basque est considérée comme devant être défendue dans la mesure où le développement du «territoire Pays Basque » passe essentiellement par l'identité basque en tant que facteur potentiellement dynamisant.

1 - Pour une étude complète de la revendication départementaliste entre la Révolution Française et la fin du $\mathrm{XX}^{\mathrm{c}}$ siècle, se reporter aux travaux de Jean-Daniel Chaussier, et notamment à son ouvrage intitulé, Quel territoire pour le Pays Basque? Les cartes de l'identité, Logiques Politiques, Éditions L'Harmattan, 1996, 295 p. 
Toutefois, dans la pratique, les objectifs de la démarche vont être à leur tour reformulés par les différentes tendances basquisantes qui vont faire du cadre partenarial de développement - le projet de «territoire Pays Basque », Lurraldea un instrument au service du projet départementaliste. De fait, à partir du milieu des années 1990, la revendication départementaliste revient au centre du débat politique local par l'intermédiaire du projet de territoire qui devait pourtant y mettre fin $^{2}$.

La démarche « Pays Basque 2010 » ne parvient donc pas à éteindre la contestation basquisante. Les abertzale et les associations départementalistes participent au nouveau cadre «territoire Pays Basque » mais restent intangibles sur la nécessité d'institutionnaliser le Pays basque nord. La mobilisation sociale est relancée par le biais de manifestations populaires, de pétitions, de consultations des élus, et de sondages. Ainsi, la cause départementaliste est progressivement perçue comme la finalité de la prospective « Pays Basque 2010 ».

Aussi, à la fin des années 1990, la position de la population et des formations politiques évolue vers plus de complexité. Le projet départementaliste a gagné en visibilité. Jusqu'ici largement opposés au projet départementaliste, les partis politiques traditionnels sont, à partir de 1999, marqués par des divisions internes que ce soit au sein du Parti Socialiste, de l'UMP (ex-RPR), ou de l'UDF qui tient la majorité au Conseil Général des Pyrénées-Atlantiques.

La revendication de création d'un Département Pays basque n'est plus aujourd'hui systématiquement considérée comme une étape vers la «construction nationale basque ». Même si les revendications soutenues par les départementalistes ne sont pas perçues comme existentielles par la majorité des habitants qui semble y adhérer, elles apparaissent désormais légitimes.

Cependant, il reste que la revendication départementaliste est portée par des acteurs différents dont les projets sont parfois contradictoires. L'ensemble de ces acteurs soutient la création d'un département mais tous n'en ont pas la même représentation : ce pluralisme idéologique est ponctuellement surmonté par un jeu d'alliances opportunistes qui illustre la légitimité du projet au sein de la classe politique locale.

\section{Octobre 1999 - Janvier 2006 : structuration de la dynamique départementaliste et naissance d'une opposition associative}

Dans ce contexte d'évolution de la revendication vers la complexité des discours et des stratégies des acteurs, il apparaît que le mouvement abertzale en particulier et le mouvement culturel basque en général, restent les moteurs de la revendication départementaliste. Les sphères basquisantes en Pays basque nord voient dans la départementalisation un moyen de répondre à leur aspiration culturelle principale, la co-officialisation de la langue basque dans la perspective de «sauver» l'euskara et, indirectement, la culture basque.

2 - Nous faisons ici référence aux travaux de Sébastien Ségas qui fait, dans sa thèse, une étude approfondie des pratiques politiques locales dans le cadre de la prospective territoriale « Pays Basque $2010 »$. In. Sébastien Ségas, La grammaire du territoire : action publique de développement et lutte politique dans les "pays", Thèse de doctorat en Science politique sous la direction de Claude Sorbets, Université Montesquieu, Bordeaux IV, 2 vol., 495 p., 2004, 159 p. d'annexes. 
Mais entre 1999 et 2003, chose nouvelle, deux formations, l'association Citoyens en Adour-Pyrénées Vivre-Ensemble et le Cercle Lissagaray sont créées et prennent l'initiative inverse en s'opposant à la revendication du Département Pays Basque. Cette apparition sur la scène publique d'associations partisanes du statu quo qui se revendiquent de la « défense de la République » modifie la teneur du débat. Durant cette période, les différentes sphères basquisantes - les élus départementalistes non-abertzale et les abertzale - maintiennent la revendication départementaliste d'actualité par l'intermédiaire de mobilisations populaires initiées par l'Appel des Cent et la «plate-forme » Batera. Lorsqu'en 2002, Batera se met en place, s'ajoutent officiellement au souhait départementaliste les revendications de création d'une Chambre d'agriculture, d'une université de plein exercice, ainsi que l'officialisation de la langue basque.

Mais jusqu'ici, les différentes tentatives n'ont reçu que des réponses négatives des pouvoirs en place. Par ailleurs, l'adhésion majoritaire des représentants politiques locaux n'apparaît véritablement qu'entre 1996 et 1999 et ne dure, du moins du point de vue de la transparence médiatique, que jusqu'à la fin de l'année 2002.

En outre, un certain nombre de phénomènes - telles la lutte armée en Espagne, la pression du gouvernement espagnol sur le gouvernement français pour ne pas favoriser, par une réforme institutionnelle, la « construction nationale basque », et la démarche Udalbiltza en tant que tentative de regroupement des élus du Pays Basque issus des sept provinces constituant Euskal Herria - desservent le projet départementaliste.

Il reste que toutes les évolutions politico-administratives mises en place localement sont considérées comme insuffisantes par la majorité des maires du Pays basque nord tant qu'elles n'atteignent pas le niveau - fantasmé ou non - de la structure départementale. La solution départementaliste est considérée comme la seule qui vaille, et, en cela, constitue le terrain privilégié de l'expression des désaccords symptomatiques du conflit local quant à la légitimité du lien entre ce qui est pensé comme relevant de la culture basque et la sphère publique.

Mais au-delà de ce panorama des pratiques politiques locales et des rapports de force contemporains, quelles sont les raisons historiques, politiques, sociologiques et idéologiques sur lesquelles bute la reconnaissance de la langue et de la culture basque en Pays basque nord?

En réponse, nous proposons une analyse des caractéristiques des différents types de représentations du lien entre domaine du politique et sphère du privé. En effet, ceux-ci induisent différentes manières de tenir compte, de reformuler ou de rejeter le socle idéologique central sous l'angle duquel le monde basque a jusqu'ici été lu : celui du mystère, de la singularité, et de l'a-temporalité . $^{3}$

Il s'avère alors nécessaire de se consacrer à l'étude des discours favorables et opposés à l'entrée dans la sphère du public de la culture et de la langue basque et à l'analyse des différentes postures à partir desquelles les acteurs vivent leurs rapports à ce qu'ils estiment relever de la culture basque. Ainsi, il apparait que les outils argumentatifs dont ils s'inspirent pour construire leur dialectique sont

3 - Nous avons entendu par l'expression monde basque, l'espace de représentations au sein duquel il est fait référence à ce qui est pensé et/ou perçu comme étant basque. 
dépendants de représentations issues des histoires institutionnelles nationales et locales dont les valeurs s'entremêlent et se disputent ou se renvoient la primauté et la responsabilité.

\section{Discours et cadre historique}

Les discours doivent alors être analysés en référence à des cadres historiques déterminés. D’abord, à partir de la Révolution française, la construction de l'Etatnation qui annonce le développement en Pays basque nord de la relation conflictuelle entre le pôle national et le pôle régional, à partir de laquelle les oppositions entre langue nationale et langue régionale, entre culture nationale et culture régionale, et, enfin, entre espace public et espace privé prennent forme. Parallèlement, il nous faut analyser le phénomène d'ethnologisation que connut le sujet Pays basque : apparaît alors l'influence passée mais aussi contemporaine de l'anthropologie en tant que discipline, dans la mesure où les acteurs, quelles que soient leurs aspirations politiques, ont recours à l'usage ou au rejet de catégories anthropologiques, telles la culture, l'identité, l'ethnie et la race.

Ainsi, par l'usage de ces notions, l'opposition au projet départementaliste rejette tout argument se revendiquant du monde basque en général, et du nationalisme basque en particulier; tandis que les différentes sphères basquisantes voient en la reconnaissance de la culture basque, par l'institutionnalisation du territoire « Pays Basque », le moyen de survie et/ou de développement de l'identité basque.

\section{La langue basque comme condensé du conflit}

La volonté de co-officialisation de la langue basque - socle de la culture basque traditionnellement soutenue par les partis nationalistes basques, ainsi que par les associations départementalistes, peut être considérée comme un thème privilégié par les acteurs et, en cela, comme le condensé du conflit. En effet, selon les associations opposées au projet territorial basque français, l'adhésion du départementalisme à la revendication de co-officialisation de l'euskara illustre le pragmatisme politique auquel seraient fidèles les abertzale, depuis la naissance de la démarche "Pays Basque 2010 ». Cette critique est justifiée par les alliances électorales entre élus de droite locaux et abertzale, avec pour fond l'idée selon laquelle le nationalisme basque et la droite traditionnelle locale auraient en commun une tendance «naturelle» au rejet des idées républicaines.

Les visées politiques nationalistes basques sont alors considérées comme nécessairement rétrogrades dans la mesure où elles seraient suscitées par la posture de défense de la culture basque considérée, par l'opposition au projet départementaliste, comme une culture étanche. C'est à cette étape du raisonnement qu'apparaît clairement l'usage de vocables incarnant le sentiment de singularité du monde basque. Cette supposée singularité, à la source de l'idée d'étanchéité, constitue le fondement même de la peur, chez nombre d'opposants, de l'institutionnalisation du Pays basque nord. L'entrée potentielle en politique de 
la culture basque, incarnée plus particulièrement dans la volonté de faire de la langue basque une langue publique, suscite des réactions de rejet.

\section{La notion d'etbnie basque comme postulat de départ}

Mais cette représentation du fait basque est née de contextes historiques déterminés. L'étude de la nature de l'héritage historiographique portant sur le sujet Pays basque illustre leur influence majeure. Après analyse, l'hypothèse d'un lien étroit entre cet héritage et l'un des référents sur lesquels s'appuient les représentations actuelles de la basquité, la croyance en l'existence de l'ethnie basque, a pu être vérifiée. Cet héritage historiographique, littéraire et scientifique, conditionne à la fois les représentations que les Basques d'aujourd'hui ont d'euxmêmes, ainsi que les représentations dont ils sont l'objet. Effectivement, l'ensemble de ce corpus tend indirectement à confirmer l'idée d'a-historicité des Basques, et contribue à perpétuer le sentiment d'immuabilité du monde basque.

Au-delà du fait que ces travaux aient été utilisés dans l'entreprise de définition de la basquité, ils encombrent aujourd'hui largement les aspirations des différentes tendances basquisantes : la revendication d'un Département Pays Basque - comme toute autre revendication institutionnelle basque - est embarrassée par cette représentation de la basquité, dominée par un imaginaire implicite assimilant identité basque et représentations essentialistes de la culture. Effectivement, ce legs littéraire et scientifique est largement instrumentalisé par l'opposition au projet dans le but de discréditer toute évolution institutionnelle qui prendrait en compte l'histoire et le présent local.

Apparait ainsi le paradigme auquel doit faire face le monde basque actuel paradigme dont le milieu du militantisme basque doit particulièrement tenir compte. Le monde basque contemporain se réfère à différents niveaux de représentation de lui-même. Il jongle entre les deux pôles de références dont il a hérité de l'Histoire. In fine, il alterne entre une représentation largement issue de la signification de l'autonyme euskalduna en tant que valeur sociale traditionnelle privilégiant l'idée d'appartenance au groupe par la langue, et les autres formes de représentations de l'identité issues de l'histoire de la construction de l'État-nation français, des premiers écrits nationalistes basques de la fin du XIX ${ }^{\mathrm{e}}$ siècle, et de la nature des discours scientifiques, encore largement diffusés et vulgarisés par les médias qui contribuent à véhiculer et à entretenir l'imaginaire d'ancestralité et de pureté du monde basque.

Le fait que la littérature n'ait longtemps su décrire ou penser le sujet Pays basque que sur le ton du mystérieux, qu'en rapport à des références explicatives relevant du mythe de l'ethnie apparaît alors comme étant à l'origine de la permanence d'une représentation essentialiste de ce qui est considéré comme relevant de l'identité basque.

Dans le cadre de notre interrogation principale, qui consiste à repérer dans quelle mesure les représentations actuelles du monde basque - et en cela leur histoire conditionnent ou non aujourd'hui la nature des discours politiques favorables ou opposés à l'institutionnalisation du Pays basque de France, ce phénomène prend toute son importance. 
L'existence d'une continuité - particulièrement du XIX siècle jusqu'à aujourd'hui - dans l'approche descriptive du Pays basque participe en effet de la fabrication du particulier, avant celle du particularisme, de l'étrange avant celle du minoritaire, puis de l'a-temporel avant celle de l'ethnique. Ce statut du monde basque apparaît comme le résultat des effets d'une représentation nationale issue de la constitution d'un académisme scientifique justifiant une hiérarchisation des cultures. La fabrication de la singularité basque, avant qu'elle ne débouche en termes contemporains sur la dénonciation de l'ethnie basque, a pour point de départ le lien historique entre la notion d'identité et le développement des sciences (particulièrement l'anthropologie, l'histoire et la biologie), dans le cadre de croyance en une corrélation Culture-Race.

Dans le contexte des débats quant à la pertinence ou non de l'institutionnalisation du Pays basque de France, il s'avère que, politiquement, cette représentation, issue de l'histoire du savoir académique national, conditionne largement la formulation des discours politiques contemporains et profite davantage à l'opposition au projet départementaliste - en tant qu'instrument de domination pour le maintien du statu quo institutionnel - qu'aux militants de l'entrée en politique de la culture basque - en tant qu'instrument de justification.

\section{La distinction Cultures à histoire/Cultures sans bistoires}

Au-delà, le développement et la permanence de cette représentation du monde basque sont intimement liés à la nature de la représentation nationale commune de la sphère du politique. Cette représentation nationale du politique est le socle à partir duquel se construisent les représentations contemporaines de ce qui apparait, dans le discours de l'opposition à la départementalisation du Pays basque nord, comme relevant de la culture basque, dite régionale ou locale.

Une logique, qui distingue en effet les cultures et/ou sociétés à progrès et les cultures et/ou sociétés pensées comme naturellement incompatibles avec toute culture politique, conditionne aujourd'hui les diverses représentations quant au rôle supposé des langues au sein du Pays Basque français : la langue française est une langue nationale et publique quand la langue basque est une langue régionale et privée.

Cette distinction est intimement liée à la construction de l'identité nationale ayant historiquement induit une tension entre un pôle de l'homogénéité et un pôle de l'hétérogénéité. Le premier établissant un lien entre le national, le cohérent et la modernité. Et le second affirmant une correspondance entre régions, traditions et origines. La rhétorique anti-départementaliste - qui s'appuie sur une représentation passéiste de la culture basque - apparaît alors comme fidèle à la typologie scientifique, d'origine évolutionniste et fonctionnaliste, opposant sociétés sans Etat et sociétés étatiques, à partir d'une grille d'analyse considérant que les sociétés ou les cultures sont des entités qui évoluent selon des mécanismes généraux analogues à ceux qui président à l'évolution des êtres vivants.

Mais la distinction sociétés historiques/sociétés sans histoire - ainsi que l'instrumentalisation idéologique de ses déclinaisons sociétés sans État/sociétés à 
État, sociétés pré-industrielles/sociétés industrielles, sociétés à écriture/sociétés sans écriture - n'a de sens qu'en tenant compte du cadre historique et idéologique particulier à partir duquel elle s'opère, à savoir la description du fait basque sur le mode de sa supposée a-historicité. L'opposition au projet départementaliste, dont le discours est issu de la formation de l'État français et de son académisme, notamment scientifique, s'appuie en effet sur une pensée hiérarchisante qui fait des cultures autochtones, dites régionales, des cultures dont les caractéristiques sont nécessairement pré-modernes et, en cela, structurellement a-politiques. Dans le cas basque, cette idée se décline jusqu'au sentiment d'a-temporalité de ce qui est considéré comme relevant de la culture locale. Ainsi, l'idéologie antidépartementaliste se réclamant des idéaux de la République est fondée sur une représentation essentialiste du fait basque. Elle développe une vision stéréotypée de ce qu'est ou doit être une culture dite régionale, ceci à partir de la fidélité à la formulation idéologique d'une norme étatique : le centralisme d'État et sa variante française, le jacobinisme.

L'effet central de cette idéologie est que s'instaure une hiérarchisation des cultures. Deux catégories apparaissent alors. La catégorie culture française est perçue comme un cadre au sein duquel une culture politique démocratique est apte à se développer. Le fait qu'une culture démocratique soit née dans le cadre de la culture française - considérée comme à vocation universelle - rend cette dernière apte au " métissage ». La puissance de cette représentation est d'autant plus étendue qu'elle évolue dans un contexte de forte croyance en la race ou, autrement dit, en référence au présupposé mythique d'une équivalence inconditionnelle entre traits de «nature » et faits de culture. La catégorie culture basque est perçue - dans le cas où l'on admet qu'elle soit une culture, ce qui n'est pas toujours de l'avis des opposants à la départementalisation - comme intrinsèquement inapte à s'inscrire dans un cadre politique. Le fait qu'elle soit pensée comme nécessairement régionale, bio-raciale, pré-moderne et a-temporelle suggère l'idée selon laquelle elle est une culture locale entière et finie, non sujette à une quelconque évolution, et en cela inapte au «métissage ».

Ainsi, la distinction entre culture légitime publique (la culture française) et culture a-légale privée (la culture basque) idéologiquement perçue comme destinée par nature à le rester, est la condition nécessaire à la formation d'un discours antidépartementaliste se réclamant de la « défense de la République».

Dans le cadre de cette distinction idéologique, l'opposition à toute revendication institutionnelle du Pays basque français fait de la question identitaire son terrain rhétorique privilégié avec l'idée, toujours sous-jacente, de l'existence d'un communautarisme, dans le sens péjoratif du terme, voire d'un racisme basque. Mais la construction de l'idée de race basque - et de son usage métaphorique actuel par l'intermédiaire de la notion d'ethnie basque - ne peut s'analyser indépendamment de l'historiographie et de l'anthropologie du XIX siècle. Cette idée d'ethnie basque constitue le socle théorique à partir duquel prend forme la distinction entre sociétés sans État (le cas basque) et sociétés à État (la République française) - et celle synonyme de sociétés sans histoire et de sociétés à bistoire, elle-même issue de l'opposition société chaude/société froide.

Dans un second temps, cette distinction est appliquée au concept de culture et débouche sur la mise en forme d'un discours ayant pour postulat de départ la nature essentialiste de ce qui relève de la culture basque. C'est cette croyance en la 
nature strictement organique du monde basque qui permet la construction du discours ayant pour but de montrer le caractère non-opérationnel de la volonté d'institutionnalisation du Pays basque nord. Cette croyance a en effet une force de persuasion très étendue dans la mesure où elle a, avant tout, statut de mythe et qu'elle est, en cela, transversale aux discours et convictions politiques. Qu'ils y adhèrent ou non, les acteurs doivent en tenir compte.

Dans ce cadre, la volonté d'inscrire la langue basque dans le domaine public - la revendication de co-officialisation - est contestée et le statut national de la langue française défendu. La détermination de faire de la langue basque, au côté de la langue française, l'une des deux langues officielles du Pays Basque nord se heurte à l'idée selon laquelle la langue nationale, langue de communication, doit être la seule à avoir une existence dans le domaine public. Une fois encore, cette idée doit être analysée dans le cadre des héritages idéologiques issus de la Révolution française et de la construction de l'identité nationale française, lesquelles donnent aux langues régionales un caractère nécessairement rural, passéiste, rétrograde, et « ethnique » dans le sens d'a-politique et en opposition au " civique ». Les caractéristiques des différentes perceptions du lien entre langue et territoire, à partir desquelles les acteurs formulent leur opposition et leur adhésion à l'entrée de la langue et de la culture basque dans le domaine public, doivent être analysées dans le cadre des effets de la distinction historique entre culture nationale et culture régionale issue de la construction de l'État-nation français, et, d'autre part, mises en lien avec les conséquences de l'ethnologisation du monde basque.

Par ailleurs, les revendications de co-officialisation de la langue basque et de création d'un Département Pays Basque suscitent des débats autour du concept de territoire et sous-entendent nécessairement l'usage de ce que nous appelons l'argument de l'bistoire. Dans ce cadre d'affrontements de discours soutenant des légitimités historiques contradictoires, les associations départementalistes ont largement moins recours à des justifications d'ordre historique que les opposants au projet. En effet, l'Histoire dont se réclament les opposants est une histoire nationale et publique et, en cela, légitime. Quant aux milieux basquisants, ils n'ont que rarement recours à ce type d'arguments très difficiles à manier puisqu'ils sont toujours potentiellement liés à l'idée de singularité basque renvoyant à une temporalité pré-moderne, si ce n'est au sentiment d'a-temporalité. Si les départementalistes s'inscrivent dans ce type d'argument, leurs opposants considèrent qu'ils se réclament d'une communauté particulière, qu'ils s'inscrivent dans la défense d'un particularisme, qu'ils sont en cela les adversaires d'une vision universelle du politique, qu'ils militent à l'encontre du sens de l'Histoire. Mais une fois de plus, ces catégories de pensée n'ont de pertinence que dans la mesure où elles s'inscrivent dans le contexte de la peur de l'ethnie basque, du fantasme de l'étanchéité de la culture basque, issue de l'ethnologisation du monde basque.

En outre, les revendications institutionnelles basques posent la question centrale de la correspondance entre identité basque et sphère publique. Dans ce cadre, les diverses représentations de l'identité qui s'expriment en Pays basque nord entrent en compétition.

L'argumentaire dont usent les opposants au projet départementaliste part alors ouvertement d'une image a-temporelle, idyllique et exotique de l'identité dite 
locale ou régionale. Compte tenu des ambiguïtés que sous-entend la diversité des représentations de la basquité - somme des traits et des représentations qualifiant l'appartenance basque -, les opposants à la création du Département Pays Basque prennent en effet le soin de privilégier ce champ de la discussion. Ils s'appuient alors indirectement sur une forme de représentation de l'identité basque qui postule une pureté première et rappellent la prédominance consensuelle de l'idée d'ethnie basque - la notion d'ethnie renvoyant ici à un sentiment d'imperméabilité totale et d'a-temporalité du groupe basque. Cette représentation niant le caractère subjectif de l'identité et la complexité des consciences basques contemporaines est en effet très utile dans l'entreprise de stigmatisation de ce qui relève du monde basque.

L'un des arguments majeurs utilisés par l'opposition au projet consiste alors à soutenir que le brassage des populations signifie la fin de la basquité et justifie le caractère non-opérationnel de la volonté d'institutionnalisation du Pays basque français. Mais cette posture dialectique signifie l'adhésion à l'argument selon lequel ne s'inscrit dans ce qui relève du monde basque que ce qui est fidèle à sa représentation ethnologisée, laquelle est largement empreinte de considérations essentialistes. Loin de réfuter «l'ethnicisme", c'est à dire la valorisation politique de l'ethnicité, qu'elle dit rejeter et combattre, cette position participe de l'ethnicisme qu'elle révoque en s'appuyant sur une représentation a-temporelle donc ethniste de l'identité adhérant ainsi à la croyance, préalablement dénoncée, en la nature essentialiste de l'ethnie basque.

Le recours aux idées polygénistes ${ }^{4}$, à partir desquelles se formule l'argumentaire de l'opposition à la départementalisation, ne fait que réitérer de façon symétrique et inverse, le modèle raciste qu'il entend dénoncer. Au-delà du fait que cette position soit paradoxale, elle participe à la stigmatisation du mouvement départementaliste. Mais une telle prise de position n'est possible que dans le cadre de la légitimité totale de la représentation nationale de l'identité instaurant une hiérarchisation entre les cultures dites régionales - pensées comme de nature strictement folklorique - et la culture nationale citoyenne.

L'idée d'une équivalence entre brassage et non-basquité est également prise en compte par une des sphères constituant le mouvement départementaliste - la sphère associative non-abertzale. Dans ce cas, l'idée est instrumentalisée dans le cadre d'une tentative de dépassement du mythe et dans l'objectif de montrer que la réforme souhaitée concerne l'ensemble des habitants.

Ces différentes postures illustrent le dilemme insoluble devant lequel se trouvent toutes revendications institutionnelles se revendiquant de la basquité. Le fait de militer pour l'entrée en politique du sujet Pays Basque débouche nécessairement sur l'accusation d'« ethnicisme ». Cependant, cette accusation s'appuie largement sur l'instrumentalisation de l'histoire de la construction de la singularité basque, dominée par l'idée d'a-temporalité et d'étrangeté intrinsèque du monde basque, avant d'être mise en compétition avec le caractère progressiste des idées issues de l'histoire politique nationale, incarné en particulier dans la référence aux Lumières.

Au-delà, ces postures instrumentalisant - dans des objectifs argumentatifs opposés - l'idée d'une équivalence entre "mélange » et non-basquité posent

4 - Nous faisons ici référence à l'idée selon laquelle les multiples dits « groupes culturels » auraient des origines déterminées et finies. 
indirectement la question de la place contemporaine de la langue basque, historiquement fondatrice de l'ethnogenèse traditionnelle. Aussi, le rapport à la langue basque entretenu par les différentes populations du Pays basque dans son ensemble, met à mal les clichés habituels sur l'existence d'un supposé nationalisme « ethnique » poussant à l'exclusion, à la xénophobie voire au racisme. Les représentations de la population en ce qui concerne la langue basque sont en effet très complexes et ne peuvent en aucun cas être traduites en une opposition catégorique entre les Basques et le reste de la population.

Il reste que l'évolution des caractéristiques linguistiques du Pays basque français, principalement marquée par le déclin prononcé de l'usage social de la langue basque - qui reste pourtant, au sein de la population, largement fondatrice de l'identité - risque de contribuer à pérenniser (sinon à développer) l'idée de singularité basque qui serait par nature inapte à entrer dans la sphère du politique. Ce déclin favorise parallèlement le développement des conditions nécessaires à la naissance d'une crispation identitaire par la marginalisation progressive et grandissante de la langue basque, jusqu'ici lien social fondamental - de fait pour les bascophones et, symboliquement, pour les non-bascophones - de la société basque.

\section{Du droit à la différence au droit à l'égalité ?}

Dans de telles circonstances, les enjeux de la reconnaissance institutionnelle et linguistique du Pays basque nord sont particulièrement conséquents. En effet, au sein de la dynamique pour la reconnaissance de la culture basque, des antagonismes idéologiques importants apparaissent. Ils se cristallisent dans la nature et le degré de reconnaissance revendiqué. Les différentes composantes du mouvement pour la reconnaissance du fait basque oscillent entre la revendication du droit à la différence et celle du droit à l'égalité.

Mais la revendication du droit à la différence s'inscrit dans la grille de lecture du monde issue de la construction de l'État-nation instaurant une hiérarchisation des cultures dites nationale et régionale. Effectivement, inscrire son argumentaire revendicatif dans l'objectif d'obtenir le droit à la différence revient à admettre comme implicites les antagonismes hiérarchisants à partir desquels le conflit se formule quels que soient les cadres thématiques - la culture, la langue, le territoire, et l'identité.

Faire de la notion de "différence » un droit signifie adhérer et légitimer l'opposition entre la sphère dite "politique » et la sphère dite « ethnique », ainsi que l'ensemble de ses dérivés - société à État/société sans État, société à écriture/société sans écriture, société historique/société sans histoire, cité/campagne,... - issus de l'opposition public/privé.

Dans le cas basque, la prégnance de ces antagonismes - constituant une vision du monde - est d'autant plus forte qu'elle a pour justification centrale la croyance, quasi consensuelle, en l'a-temporalité du monde basque, la croyance en l'existence de l'ethnie basque, catégorie anthropologique née au XIX ${ }^{\mathrm{e}}$ siècle. Ainsi, cette 
revendication du droit à la différence est nécessairement empreinte des représentations classiques de ce à quoi doit correspondre une culture régionale.

Au contraire, la revendication du droit à l'égalité penche pour un traitement équitable de ce qui est considéré comme relevant de la culture basque, cette équité passant par l'entrée dans la sphère publique, par la création d'un «toit politique». Cette revendication du droit à l'égalité s'appuie sur une perception opposée aux visions conservatrices et patrimoniales du fait culturel basque, rejetant les représentations régionales folklorisantes de l'identité, et en rupture avec l'idée d'ethnie toujours prégnante dans la représentation collective dominante du fait de son statut de mythe.

\section{L'avenir de la culture basque en suspens}

L'étude des revendications institutionnelles basques illustre de manière exemplaire la difficulté de raisonner au-delà d'une vision essentialiste du fait culturel en général et du fait culturel basque en particulier. Il apparaît qu'à terme cette difficulté sera partie prenante des phénomènes allant à l'encontre de la survie de la culture basque dans le contexte de sa non-reconnaissance. En effet, les explications d'ordre sociologique ne sont pas nécessairement significatives de la nature des prises de position quant à la reconnaissance de la culture basque. L'élément le plus influent, justifiant systématiquement l'adhésion (ou non) à l'entrée en politique de la culture basque, est le rapport que l'individu entretient au mythe, l'adhésion ou le rejet de celui-ci, ou l'alternance entre les deux postures.

Ainsi, les mouvements basques, qu'ils soient abertzale ou strictement départementalistes et, au-delà, le monde basque en général, subissent plus qu'ils ne revendiquent le mythe de la communauté d'origine et ses dérivés tels que l'ethnie. Le mouvement abertzale - tout comme l'ensemble des autres tendances politiques représentées en Pays basque nord - n'est pas uni derrière une seule et même représentation de l'identité basque mais il est l'unique force politique au sein de laquelle une tendance à défendre et à développer une conception dynamique de l'identité basque existe dans la mesure où son discours revendique un lien potentiel entre citoyenneté et culture basque. Il tente ainsi de tempérer les visions essentialistes de la basquité issues de l'ethnologisation du monde basque dans le cadre de la naissance de l'État en tant que structure politique génératrice et institutrice de représentations régionales du fait culturel. En Pays basque nord, il propose un compromis : celui d'inscrire la culture basque dans la sphère du public tout en ne reniant pas les représentations régionales actuelles dominantes de la culture basque - qui relèvent de l'affect - issues du mythe de la singularité basque et considérant comme implicite le lien entre identité basque et conditions identitaires héritées.

Nous avons proposé une grille d'analyse partant du postulat qu'associé à sa noninstitutionnalisation et à la perte constante de l'usage social de la langue basque, le traitement littéraire et scientifique sous le mode de l'a-temporalité de ce qui relève ou paraît relever du monde basque avait abouti à une tendance à lire le monde social basque sous le spectre de l'essentialisme. 
Ainsi, la survie de ce qui est considéré comme relevant de la culture basque est prisonnière de l'opposition nationale/régionale et dépendante de la pérennité ou non de celle-ci. En effet, cette opposition induit nécessairement une représentation essentialiste de ce qui est considéré comme relevant de la culture régionale, pensée comme fondée en nature dans le cadre de la grille de lecture du monde établissant un lien intrinsèque et indépassable entre faits de culture et faits de nature. Aussi, les mouvements de reconnaissance du fait basque en France ne participent pas du phénomène d'ethnicisation de la société. Une part importante de la classe politique et de la société civile participe au contraire à une tentative interne de contestation des effets néfastes du statut régional du monde basque et milite pour le désenclavement de la culture basque de l'imaginaire constituant le mythe de l'etbnie basque.

Les mouvements pour la reconnaissance du sujet Pays basque français, dans leur tentative de relecture du monde basque, ont l'avantage de ne pas avoir à rompre avec la cosmogonie basque traditionnelle dans leur démarche de construction cohérente du territoire Pays basque : ils revendiquent essentiellement, du point de vue culturel comme symbolique, la légitimité politique non-exclusive de l'autonyme euskalduna, référent à la fois historique et privé, institué et public.

Cette étude du cas basque participe d'une question qui la dépasse : dans quelle mesure les différentes dynamiques se revendiquant de la notion d'identité sont aptes à rompre avec le rapport fondateur les ayant historiquement constitué, à savoir la relation nature-culture?

Cette question, dans le cadre de la construction européenne et du processus de globalisation, s'avère centrale dans la mesure où une gestion politique apaisée de la diversité culturelle ne passera que par le dépassement du présupposé selon lequel l'origine des hommes détermine nécessairement et inconditionnellement leur nature, comprise comme synonyme de leur culture. 